\title{
Logging Multi-Component Supply Chain Production in Blockchain
}

\author{
Ivan Chistiakov \\ Faculty of Computer Science, \\ National Research University Higher \\ School of Economics
}

\author{
Yash Madhwal \\ Center for Computational and \\ Data-Intensive Science and \\ Engineering, Skolkovo Institute of \\ Science and Technology
}

\author{
Yury Yanovich \\ Center for Computational and \\ Data-Intensive Science and \\ Engineering, Skolkovo Institute of \\ Science and Technology and \\ Laboratory of Data Mining and \\ Predictive Modelling Institute for \\ Information Transmission Problems
}

\begin{abstract}
The supply chain is a thriving industry where numerous parties have different interests. Subsequently, the immense volume of data produced is difficult to audit. Some information can be lost or intentionally distorted in the process. Blockchain as an open, public, borderless, neutral, and censorship-resistant architecture can significantly complement supply chains. A new supply chain architecture is proposed in this work, where the tokenized directed acyclic hypergraph (DAG) represents real-world production processes. An anti-aerosol respirator manufacturing is used as an illustration example. By tokenizing all parts of multi-component products, supply chain data is automatically timestamped and secured. Moreover, the DAG design allows one to trace-back all the elements of the final product to their origin. Blockchain can formally audit the entire supply chain without the need to go from place to place. A single incorruptible operations log creates an enabling environment for an unbiased reputation system to emerge.
\end{abstract}

\section{CCS CONCEPTS}

- Information systems; • Information systems applications; - Enterprise information systems; • Enterprise applications; - Computer systems organization; - Architectures; - Distributed architectures; $\bullet$ Peer-to-peer architectures;

\section{KEYWORDS}

Blockchain, supply chain, smart contract, directed acyclic hypergraph

\section{ACM Reference Format:}

Ivan Chistiakov, Yash Madhwal, and Yury Yanovich. 2021. Logging MultiComponent Supply Chain Production in Blockchain. In 2021 The 4th International Conference on Computers in Management and Business (ICCMB 2021), January 30-February 01, 2021, Singapore, Singapore. ACM, New York, NY, USA, 6 pages. https://doi.org/10.1145/3450588.3450604

Permission to make digital or hard copies of all or part of this work for personal or classroom use is granted without fee provided that copies are not made or distributed for profit or commercial advantage and that copies bear this notice and the full citation on the first page. Copyrights for components of this work owned by others than ACM must be honored. Abstracting with credit is permitted. To copy otherwise, or republish, to post on servers or to redistribute to lists, requires prior specific permission and/or a fee. Request permissions from permissions@acm.org.

ICCMB 2021, fanuary 30-February 01, 2021, Singapore, Singapore

(C) 2021 Association for Computing Machinery.

ACM ISBN 978-1-4503-8861-0/21/02 . \$ \$15.00

https://doi.org/10.1145/3450588.3450604

\section{INTRODUCTION}

The supply chain industry's growth globally enabled the quality manufacturing of products [1]. Over the decades, supply chain networks have evolved into a more complex and complicated manufacturing process manufacturing complex and composite products. Consumer goods, such as clothes, food, furniture, etc., go through multiple intermediaries layers of the supply chain until the retailer. An average supply chain comprises producers, vendors, warehouses, transportation companies, distribution centres, and retailers.

Loss of information is inevitable and lacks trust, thus leading to an inefficient supply chain's functioning. At the same time, the end customer has no option but to rely on its authenticity. Another problem in the modern supply chain industry is the high entry threshold for new entities like large manufacturers willing to work with established companies. All these problems narrow down to trust between entities.

With the beginning of Bitcoin [2], it became possible to transfer digital value with unprecedented transparency and security. Blockchain-the underlying technology of Bitcoin, guarantees data safety and availability as a single source of information. The blockchain architecture's openness allows anyone to verify integrity and validity [3], [4]. The alternate cryptocurrency, Ethereum [5], enables the transfer of digital value more flexibly with smart contracts.

This paper proposes a supply chain architecture in which the blockchain is a single secure bulletin board. This information source is neutral and incorruptible and allows participants to reach a consensus effectively. Since all information is available in one place, checking the reliability of counterparties is much more comfortable. At the same time, the final buyer can independently verify the quality of the received product.

\section{RELATED WORK}

Lack of visibility in the product's tracking results in counterfeiting or replacement of products. Protecting legitimate supply chains from fraud has become a significant challenge [6]- [8]. Some companies are trying to develop highly sophisticated methods that would be practically impossible to falsify or even reproduce. Some manufacturers choose to conceal specific details according to which they can check their parts' authenticity in the market. These costs come from many areas, and reducing the lack of visibility and accountability can increase operational expenses. 
Businesses adopt blockchain [2] over the existing database storage system because it provides data integrity and cannot be altered, making it easy to audit and track [3], [4]. Some studies have provided theoretical and empirical studies on blockchain traceability and tracking capability gaining customer confidence [9]- [15].

The common way to launch a blockchain on the top of the existing supply chain is to perform tokenization-asset conversion into the blockchain where it can be recorded, stored or transferred [16]. One needs to associate products with(non-fungible, utility) tokens and commit their movement and transformation history to the ledger. The project also needs currency tokens (fungible) if the payment logic is assumed to be performed on the blockchain. As a result, companies can manage the flow of goods with blockchain's potentiality and simultaneously monitor financial transactions. The majority of the blockchain application with supply chain methodology focuses on tracking a product's physical movement instead of its production journey [17]- [28]. Labels design and choice [29], [30], together with the privacy aspects [31], are under discussion as well. Big companies also present their solutions for the supply chain but usually without a technical description and open-source code. Thus, Provenance implemented this concept for tuna fish, where the source of fish's origin could be tracked from port to plate [32]. Walmart and FedEx have integrated blockchain with the supply chain in tracking a single product type [33], [34], e.g., to monitor its physical position, overlooking conversion or transformation of the product into a different form.

The best practice for blockchain tokenization is its expression as an open-source smart contract with formally declared emission, transfer, and transformation rules [5], [35]. A solidity programming language is a common tool for smart contract implementation due to the big community and standards presence [16]. Most of the standards are proposed as Ethereum Request for Comments (ERCs) and include currency, utility, security token types, and hybrid versions [36]- [39]. Writing data of a specific tokenized product on the blockchain is not challenging. However, committing the data of merging two or more non-fungible token and convert it to form a new token still problematic. Although ERC1155 [38] tokens give the leverage of combining tokens to different indexes, they are not considered a new token type because these tokens are liable to move or be spent. The manufacturing process can be regarded as a directed acyclic hypergraph (DAG) [40], where vertices represent product-state pairs.

Edges are the transitions from a subset of vertices to another subset of vertices. For example, one can use a 1-to-1 transaction to represent selling and a 2-to-1 transaction to represent an assembling of two details into a new one. Hypergraphs are used instead of graphs to provide events atomicity by design. We consider a multicomponent production logging in blockchain and propose a token standard to deal with it. Information about all processes occurring in supply chains should be structured and ordered naturally and written in the blockchain. It is necessary to provide a way to process and log all possible actions in supply chains. Papers [41]- [43] deal with the same problem. Our impact is the implementation within a single token standard and hypergraphs with logging and atomicity by design. The proposed standard is an extension on the top of ERC1155.

\section{METHODOLOGY}

Processes inside the supply chain network need to be recorded to the blockchain in a structured and ordered manner. In parallel, to provide a way to process and log all plausible actions in a real supply chain model.

\subsection{Supply chain directed acyclic hypergraph}

We formalized a methodology by considering an arbitrary supply chain as a directed acyclic hypergraph. Graph Vertex represents the state of a product in a supply chain at a specific location in the process, i.e., product-state pair. Graph Edge is the transition of a product-state pair to another product-state pair, as shown in Figure 1

If multiple products are used to create another composite product, the node would have multiple sources, indicating the part it comprises. If the composite product has been disassembled, then the edge would have multiple target vertices for the resulting parts. We assume that if a product in the supply chain has changed its state once, it cannot return to its previous state (tokens are non-fungible by default), but it can change to a similar state as in the previous one. Such that there are no directed cycles in the graph.

\subsection{Standard of tokens}

Token standards define as a common interface for smart contracts to unify and simplify the integration process for var-ious wallets, exchanges, and other services. Token standards distinguish between interchangeable, non-interchangeable, and hybrid tokens:

- Interchangeable tokens are fungible tokens, i.e., they do not differ from each other. For example, if one token represents a value, then it will be equal to any other token[36].

- Non-interchangeable tokens are non-fungible tokens, i.e., they differ from each other. For example, for a tokenized real estate, one token that represents a specific real estate object will not be equal to any other token. Each token stands unique [37].

- Hybrid tokens are a combination of fungible and nonfungible tokens, i.e., creating sets of interchangeable tokens. For example, tokenizing investment portfolios, where each investment portfolio can contain different sets of fungible and non-fungible token sets [38], [39].

We use the ERC1155 token for our implementation, a standard for hybrid tokens that can work within a single smart contract. A smart contract deployed by a company's ethereum address can create tokens, make token transactions, and perform other functionalities as long as they are the token owner.

This approach saves resources and reduces commissions and is also ideal for tokenizing supply chain models because a company can produce different products. Therefore, to represent it in the tokenized standard, it will work best using the ERC1155 standard.

\section{IMPLEMENTATION}

A product goes through different supply chain entities, and each entity has different business processes and agreements among different entities. Each entity should deploy its smart contract. Such a smart contract will manage tokens representing the supply chain 


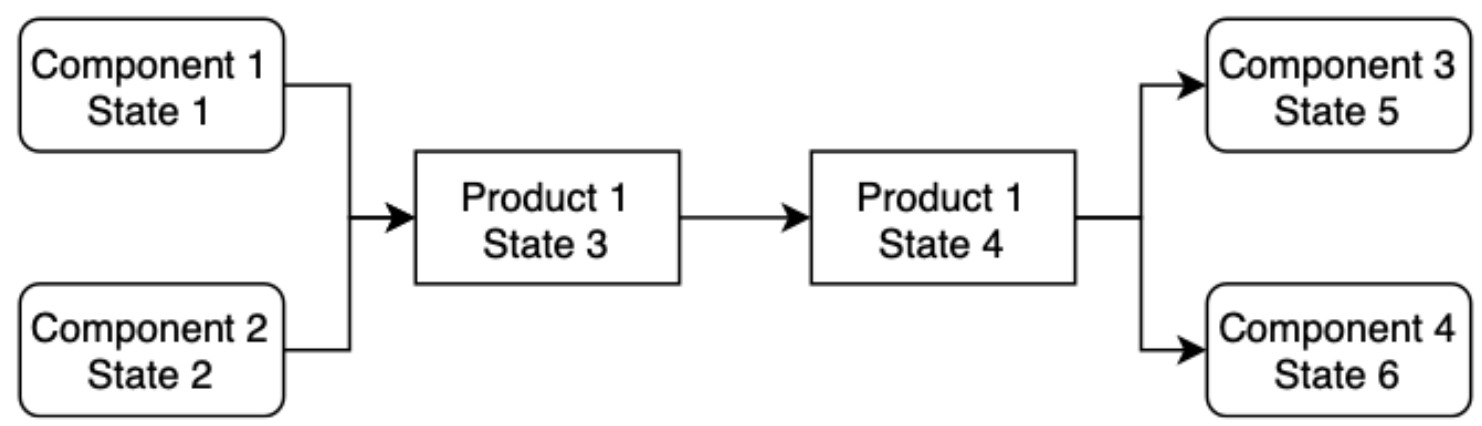

Figure 1: Example of directed acyclic hypergraph for supply chain.

products in the entities' possession. The smart contract also handles all manipulations that occur with these tokens, i.e., products.

If the product-state pair changes, the company creates a new token. Such product-state pair transition can be represented as vertices and edges on the supply chain network. The supply chain's significance is that different entities own the product at a different time. On the blockchain, various smart contracts and edges must point to tokens transfer from other smart contracts. Simultaneously, designing such edges within the ERC1155 standard framework is designed only for sending tokens to another account existing in the same smart contract.

\subsection{Token address as vertex}

A token is a non-interchangeable token or a set of interchangeable tokens that exits at different indexes independent of each other. Each created token is assigned a unique address such that another token can be sent to it, hence creating the necessary edge between two vertices. If the tokens are located in different smart contracts, a token is created in the receiver's smart contract, and then the transaction to the created token address is done in the sender's smart contract. The operation of sending a token is inexpensive because changes occur in two memory cells, i.e., in the sender's balance and the recipient's balance.

For the supply chain graph's data to be consistent, the private key should not exist to the token address. The token addresses must be unique. The smart contract's unique address can be used to get the next token address, thus increasing it in token creation.

$$
\text { token Address }=\text { contract Addr + token Index Value. }
$$

The cryptographic hash function calculates the smart contract addresses. Therefore, it is impossible to deploy the smart contract to the desired address. Consequently, you can send tokens to "neighbouring" smart contract addresses by permanently blocking these tokens and creating edges in the graph. Since all addresses are indexed, the token address is the "neighbour" address of the corresponding smart contract and can quickly restore one address by knowing the other.

\subsection{Address of the set of tokens}

For splitting a single token into multiple tokens, the preceding method is inefficient. The tokens will be blocked once transacted and will have zero balance to create a second edge. The set of tokens' address is to generalize the technique described above with multiple outgoing edges.

Formalizing an address represents a set of outgoing addresses by sequentially indexing the receiving address in an array. The token set's desired address is the bitwise exclusive "or" address of the smart contract and the hash of the set of token numbers.

$$
\text { tokenSetAddress }=\text { contractAddr } \oplus \text { hash (tokenArray). }
$$

Because of the cryptographic hash function, it is impossible to recover any information from the address of a set of tokens. Therefore, a set of token addresses should be recorded to the blockchain before using the set of tokens' address.

\subsection{ERC1155 DAG}

The basic implementation of the ERC1155 exits and is inherited for generating a smart contract for the company. The most suitable smart contract option is the ERC1155Mintable, in which token issuance is unlimited, and supply chain participants can create various new tokens as needed. As per the requirement, each entity can deploy an instance of the smart contract and function independently. The contract owner can only create tokens with balance and transfer to the calculated token address whose private key doesn't exist, hence making a transaction on the blockchain. Once a final product is created, the product owner also owns an instance of the contract and can quickly transfer the ownership.

\section{EXAMPLE}

The implementation is considered for a simplified example of an anti-aerosol respirator's supply chain. Three entities are involved in manufacturing anti-aerosol respirators. First, the respirator manufacturer produces gas mask respirators, which are produced from plastic pellets. The plastic pellets take different forms until gas mask respirators are manufactured, in the later stage, used by anti-aerosol manufacturers. Second, the filter manufacturers produce and manage the supply chain of two different types of filters, i.e., gas mask filter and anti-aerosol filter, procured by respirator manufacturer and anti-aerosol manufacturer. Third, the anti-aerosol manufacturer procures the gas mask respirators and replaces the gas mask filter with anti-aerosol filters procured from filter manufacturers to generate anti-aerosol respirators. The respirator store regularly buys gas masks for subsequent retail sale. 


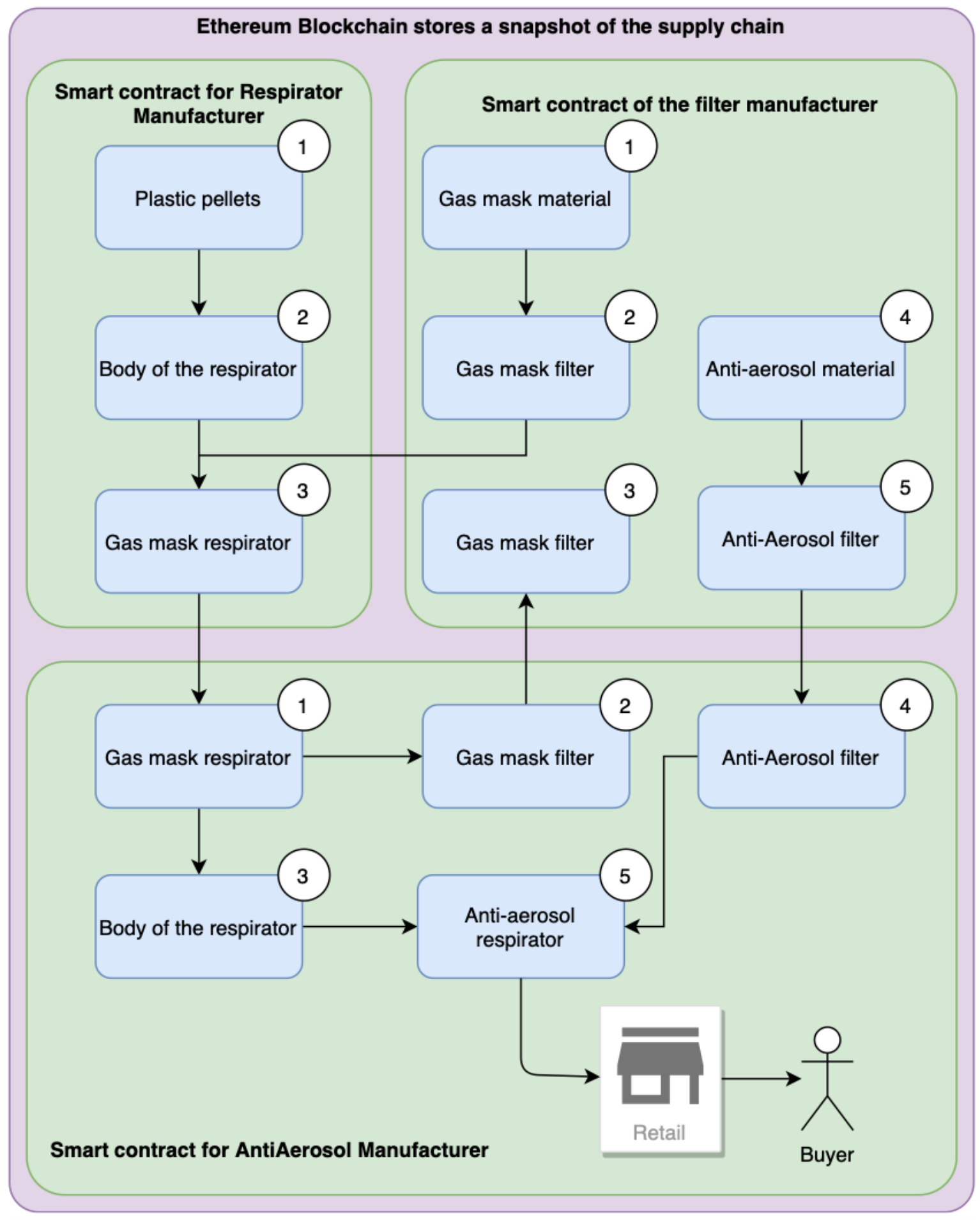

Figure 2: Simplified supply chain for Anti-aerosol respirator Manufacturer 
An illustration of one of these anti-aerosol respirators' supply chains in terms of smart contracts and ERC1155DAG tokens is provided in Figure 2. Three instances of the smart contract were deployed ganache test network to reproduce the actions from the example. Ganache is a prototype of a blockchain that runs on a personal computer, it is mainly used for developing a decentralised application (DApp). In order to distinguish between these contracts, additional contract ERC1155SUPPLYCHAINCOMPANY was deployed, which will store the company's name that owns a particular instance of the contract.

The demo of the contract is available at https://github.com/ yashmadhwal/SupplyChainCase.

\section{CONCLUSION}

The proposed architecture protects buyers from counterfeit products and gives the buyer a snapshot of the entire product supply chain. The information is organized chronologically, forming a directed acyclic graph of tokens. Such structured data is easily analyzed and verified, both internally and by the end-user. With all information available on the blockchain, verification and auditing are feasible. With the complete tokenization of products in supply chains and the use of a single source of information, i.e., blockchain can be relied on for data integrity and reliability. The abundance of data in a single source of information will allow tracking disputes and assessing the reliability of contractors effectively.

\section{REFERENCES}

[1] D. Simchi-Levi, E. Simchi-Levi, and P. Kaminsky, Designing and Managing the Supply Chain: concepts, strategies, and case studies. McGraw-Hill/Irwin, 2003.

[2] S.Nakamoto,"Bitcoin:APeer-to-PeerElectronicCashSystem,"www.bitcoin.org, pp 1-9, 2008. [Online]. Available: https://bitcoin.org/bitcoin.pdf

[3] V. Buterin, "On Public and Private Blockchains - Ethereum Blog,"2015. [Online]. Available: https://blog.ethereum.org/2015/08/07/on-public-and-privateblockchains/

[4] Bitfury Group, “On Blockchain Auditability,”bitfury.com, pp. 1-40,2016. [Online]. Available: https://bitfury.com/content/downloads/bitfury-white-pape r-onblockchain-auditability.pdf

[5] V. Buterin,"Ethereum White Paper: A Next Generation Smart Contract \& Decentralized Application Platform," Etherum, pp.1-36, 2014. [Online]. Available: https://github.com/ethereum/wiki/wiki/White-Paper

[6] F. El-Jardali, E. A. Akl, R. Fadlallah, S. Oliver, N. Saleh, L. El-Bawab,R. Rizk, A. Farha, and R. Hamra, "Interventions to combat or prevent drug counterfeiting: a systematic review," BMJ Open, vol. 5,no. 3, pp. e006 290.01-e006 290.11, 32015. [Online]. Available:https://bmjopen.bmj.com/lookup/doi/10.1136/bmjopen-2014006290

[7] F. R. P. d. Lima, A. L. Da Silva, M. Godinho Filho, andE. M. Dias, "Systematic review: resilience enablers to combat counterfeit medicines," Supply Chain Management: An International Journal, vol. 12, no. 3, pp. 117-135, 32018. [Online] Available: https://www.emerald.com/insight/content/doi/10.1108/SCM-04-20170155/full/html

[8] Di Liddo, "Counterfeiting Models: Mathematical/Economic," in Encyclopedia of Law and Economics. New York, NY: Springer New York, 2019, pp. 418-422.

[9] E. Ben-Sasson, A. Chiesa, C. Garman, M. Green, I. Miers, E. Tromer,M. Virza, E. B. Sasson, A. Chiesa, C. Garman, M. Green, I. Miers,E. Tromer, and M. Virza, "Zerocash: Practical Decentralized Anonymous E-Cash from Bitcoin," in Proceedings of the 2014 IEEE Symposium onSecurity and Privacy. IEEE, 5 2014, pp. 459-474.

[10] B. Bunz, J. Bootle, D. Boneh, A. Poelstra, P. Wuille, and G. Maxwell, "Bulletproofs: Short Proofs for Confidential Transactions and More," in 2018 IEEE Symposium on Security and Privacy (SP), vol. 2018-May.IEEE, 5 2018, pp. 315-334.

[11] D. Korepanova, M. Nosyk, A. Ostrovsky, and Y. Yanovich, "Building a Private Currency Service Using Exonum," in 2019 IEEE International Black Sea Conference on Communications and Networking (BlackSeaCom). IEEE, 6 2019, pp. 1-3. [Online]. Available: https://ieeexplore.ieee.org/document/8812875/

[12] S. Abramova, P. Sch öttle, and R. B öhme, "Mixing Coins of DifferentQuality: A Game-Theoretic Approach," inLecture Notes in ComputerScience (including subseries Lecture Notes in Artificial Intelligence andLecture Notes in Bioinformatics), vol. 10323 LNCS.Springer, Cham,4 2017, pp. 280-297.
[13] Y. Yanovich, P. Mischenko, and A. Ostrovskiy, "Shared Send Untangling in Bitcoin," bitfury.com, vol. 2016, pp. 1-25, 2016.

[14] D. Ermilov, M. Panov, and Y. Yanovich, "Automatic Bitcoin Address Clustering," in2017 16th IEEE International Conference on Machine Learning and Applications (ICMLA).IEEE, 12 2017, pp. 461-466.[Online]. Available: http://ieeexplore.ieee. org/document/8260674/

[15] S. S. Chawathe, "Clustering Blockchain Data." Springer, Cham, 2019,pp. 43-72.

[16] M. Shirole, M. Darisi, and S. Bhirud, "Cryptocurrency Token: An Overview." Springer, Singapore, 2020, pp. 133-140.

[17] D. Mohanty and D. Mohanty, "Supply Chain-Gold Tokenization," in R3 Corda for Architects and Developers. Apress, 2019, pp. 193-198.

[18] S. A. Abeyratne and R. P. Monfared, "Blockchain ready manufacturing supply chain using distributed ledger," International Journal of Research in Engineering and Technology, vol. 05, no. 09, pp. 1-10, 92016.

[19] Y. Madhwal and P. Panfilov, "Blockchain And Supply Chain Management: Aircrafts' Parts' Business Case," in Annals of DAAAM and Proceedings of the International DAAAM Symposium, 2017, pp. 1051-1056.

[20] P. Kostyuk, S. Kudryashov, Y. Madhwal, I. Maslov, V. Tkachenko, and Y. Yanovich, "Blockchain-Based Solution to Prevent Plastic PipesFraud," in 2020 Seventh International Conference on Software Defined Systems (SDS).IEEE, 4 2020, pp. 208-213. [Online]. Available: https://ieeexplore.ieee.org/document/9143879/

[21] S. Kudryashov, S. Kruglik, I. Maslov, and Y. Yanovich, "Supply-Chain Management System for Plastic Pipes Market Based on Open Blockchain Framework," in2020 IEEE 21st International Symposium on "A World of Wireless, Mobile and Multimedia Networks"(WoWMoM).IEEE, 8 2020, pp. 188-190. [Online]. Available: https://ieeexplore.ieee.org/document/9217641/

[22] Y. Yanovich, I. Shiyanov, T. Myaldzin, I. Prokhorov, D. Korepanova, and S. Vorobyov, "Blockchain-Based Supply Chain for Postage Stamps,"Informatics, vol. 5, no. 4, p. 42, 112018.

[23] D. Korepanova, S. Kruglik, Y. Madhwal, T. Myaldzin, I. Prokhorov, I.Shiyanov, S.Vorobyov, and Y.Yanovich, "Blockchain-Based Solution to Prevent Postage Stamps Fraud," in2019IEEE International Conference on Blockchain and Cryptocurrency (ICBC). IEEE, 2019, pp. 171-175. [Online]. Available: https://ieeexplore. ieee.org/document/8751495/

[24] S. Malik, V. Dedeoglu, S. S. Kanhere, and R. Jurdak, "TrustChain:Trust Management in Blockchain and IoT Supported Supply Chains," in 2019 IEEE International Conference on Blockchain (Blockchain).IEEE, 7 2019, pp. 184-193. [Online]. Available: https://ieeexplore.ieee.org/document/8946187/

[25] E. Balistri, F. Casellato, C. Giannelli, R. Lazzarini, C. F. Ngatcha Keyi, and C. Stefanelli, "Servitization in the Era of Blockchain: the Ice Cream Supply Chain Business Case," in2020 International Conference on Technology and Entrepreneurship (ICTE).IEEE, 9 2020, pp. 1-8.[Online]. Available: https://ieeexplore.ieee.org/ document/9215539/

[26] S. Pearson, D. May, G. Leontidis, M. Swainson, S. Brewer, L. Bidaut,J. G. Frey, G. Parr, R. Maull, and A. Zisman, "Are Distributed Ledger Technologies the panacea for food traceability?" Global Food Security, vol. 20, pp. 145-149, 3 2019. [Online]. Available: https://linkinghub.elsevier.com/retrieve/pii/S2211912418301408

[27] J. G. C. Neto, R. C. Barbosa, F. C. H. Marino, and N. L.Zanutim, "Prevention of Medication Loss through a Marketplaceand Blockchain," inProceedings of the 2019 2nd International Conference on Blockchain Technology and Applications. New York, NY, USA: ACM, 12 2019, pp. 124-128. [Online]. Available:https://dl. acm.org/doi/10.1145/3376044.3376059

[28] Y. Madhwal, "Implementation of Tokenised Supply Chain UsingBlockchain Technology," in2020 IEEE 21st International Symposium on "A World of Wireless, Mobile and Multimedia Networks"(WoWMoM).IEEE, 8 2020, pp. 66-67. [Online]. Available: https://ieeexplore.ieee.org/document/9217696/

[29] Feng Tian, "An agri-food supply chain traceability system for China based on RFID \& blockchain technology," in201613th International Conference on Service Systems and Service Management (ICSSSM).IEEE, 6 2016, pp. 1-6. [Online]. Available: http://ieeexplore.ieee.org/document/7538424/

[30] N. Alzahrani and N. Bulusu, "Block-Supply Chain: A New Anti-Counterfeiting Supply Chain Using NFC and Blockchain," in Proceedings of the 1st Workshop on Cryptocurrencies and Blockchains for Distributed Systems - CryBlock'18. New York, New York, USA: ACMPress, 2018, pp. 30-35.

[31] H. Wu, Z. Li, B. King, Z. Ben Miled, J. Wassick, and J. Tazelaar, "A Distributed Ledger for Supply Chain Physical Distribution Visibility,"Information, vol. 8, no. 4, p. 137, 11 2017. [Online]. Available: http://www.mdpi.com/2078-2489/8/4/137

[32] Boulais,"ExploringProvenanceofTunausingDistributedLedgers,"Tech.Rep.,2019.[Online].Available:https://viral.media.mit. edu/pub/tunaprovenance/release/1

[33] B. Tan, J. Yan, S. Chen, and X. Liu, "The Impact of Blockchain on Food Supply Chain: The Case of Walmart," in Lecture Notes in Computer Science (including subseries Lecture Notes in Artificial Intelligence andLecture Notes in Bioinformatics). Springer Verlag, 12 2018, vol. 11373LNCS, pp. 167-177.

[34] IEEE Innovation at Work, "Shipping Giants Employ Blockchain Technology to Manage Supply Chain Logistics,"2018.[Online]. Available: https://innovationatwork.ieee.org/shipping-giants-employ-blockchaintechnology-to-manage-supply-chain-logistics/ 
[35] M. Bartoletti and L. Pompianu, "An Empirical Analysis of Smart Contracts: Platforms, Applications, and Design Patterns," 2017, vol.10323 LNCS, pp. 494-509.

[36] F.VogelstellerandV.Buterin,"ERC-20TokenStandard,"2015.[Online].Available:https: //github.com/ethereum/EIPs/blob/master/EIPS/eip-20.md

[37] ERC-721, "ERC-721," 2018. [Online]. Available: http://erc721.org/

[38] W. Radomski, A. Cooke, P. Castonguay, J. Therien, E. Binet, andR. Sandford, "ERC1155: Multi Token Standard." [Online]. Available:https://github.com/ ethereum/EIPs/issues/1155

[39] V.Davydov, A.Gazaryan, Y.Madhwal, and Y.Yanovich, "Token Standard for Heterogeneous Assets Digitization into Commodity," in Proceedings of the 2019 2nd International Conference on Blockchain Technology and Applications. NewYork, NY, USA: ACM, 12 2019, pp. 43-47. [Online]. Available:https://dl.acm.org/doi/10. $1145 / 3376044.3376053$
[40] G. Ausiello and L. Laura, "Directed hypergraphs: Introductionand fundamental algorithms-A survey," Theoretical Computer Science, vol. 658, pp. 293306, 1 2017. [Online]. Available:https://linkinghub.elsevier.com/retrieve/pii/ S0304397516002097

[41] H. M. Kim and M. Laskowski, "Towards an Ontology-Driven Blockchain Design for Supply Chain Provenance," SSRN Electronic Journal, vol. 25, no. 1, pp. 18-27, 8 2016. [Online]. Available:http://www.ssrn.com/abstract $=2828369$

[42] M. Westerkamp, F. Victor, and A. K üpper, "Blockchain-based Supply Chain Traceability: Token Recipes model Manufacturing Processes,"2018. [Online]. Available: https://doi.org/10.14279/depositonce-7295http://arxiv.org/abs/1810.09843

[43] M. Westerkamp, F. Victor, and A. Kupper, "Tracing manufacturing processes using blockchain-based token compositions," Digital Communications and Networks, vol. 6, no. 2, pp. 167-176, 52020. [Online]. Available: https://linkinghub.elsevier. com/retrieve/pii/S235286481830244X 